\title{
Strong convergence theorems of nonlinear operator equations for countable family of multi- valued total quasi- $\varphi$-asymptotically nonexpansive mappings with applications
}

\author{
Shih-Sen Chang ${ }^{1 *}$, Lin Wang ${ }^{1}$, Yong-Kun Tang ${ }^{1}$, Yun-He Zhao ${ }^{1}$ and Zao-Li Ma ${ }^{2}$
}

\footnotetext{
* Correspondence: changss@yahoo. $\mathrm{cn}$

${ }^{1}$ College of Statistics and Mathematics, Yunnan University of Finance and Economics, Kunming, Yunnan 650221, China

Full list of author information is available at the end of the article
}

\begin{abstract}
The purpose of this article is first to introduce the concept of total quasi- $\varphi$ asymptotically nonexpansive multi-valued mapping which contains many kinds of mappings as its special cases, and then by using the hybrid shrinking technique to propose an iterative algorithm for finding a common element of the set of solutions for a generalized mixed equilibrium problem, the set of solutions for variational inequality problems, and the set of common fixed points for a countable family of multi-valued total quasi- $\varphi$-asymptotically nonexpansive mappings in a real uniformly smooth and strictly convex Banach space with Kadec-Klee property. The results presented in the article not only generalize some recent results from single-valued mappings to multi-valued mappings, but also improve and extend the main results of Homaeipour and Razani.

2000 AMS Subject Classification: 47J06; 47J25.

Keywords: multi-valued total quasi- $\varphi$-asymptotically nonexpansive mappings, quasi$\varphi$-asymptotically nonexpansive multi-valued mappings, quasi- $\varphi$-nonexpansive multivalued mappings, relatively nonexpansive multi-valued mappings, generalized projection
\end{abstract}

\section{Introduction}

Throughout this article, we always assume that $X$ is a real Banach space with the dual $X^{*}, C$ is a nonempty closed convex subset of $X$, and $J: X \rightarrow 2^{X}$ is the normalized duality mapping defined by

$$
J(x)=\left\{f^{*} \in X^{*}:\left\langle x, f^{*}\right\rangle=\|x\|^{2}=\left\|f^{*}\right\|^{2}\right\}, x \in E .
$$

In the sequel, we use $F(T)$ to denote the set of fixed points of a mapping $T$, and use $\mathscr{R}$ and $\mathscr{R}^{+}$to denote the set of all real numbers and the set of all nonnegative real numbers, respectively. We denote by $x_{n} \rightarrow x$ and $x_{n} \rightarrow x$ the strong convergence and weak convergence of a sequence $\left\{x_{n}\right\}$, respectively.

Let $\Theta: C \times C \rightarrow \mathscr{R}$ be a bifunction, $\psi: C \rightarrow \mathscr{R}$ be a real valued function, and $A$ : $C \rightarrow X^{*}$ be a nonlinear mapping. The so-called generalized mixed equilibrium problem is to find $u \in C$ such that

(C) 2012 chang et al; licensee Springer. This is an Open Access article distributed under the terms of the Creative Commons Attribution License (http://creativecommons.org/licenses/by/2.0), which permits unrestricted use, distribution, and reproduction in any medium, provided the original work is properly cited. 


$$
\Theta(u, y)+\langle A u, y-u\rangle+\psi(\gamma)-\psi(u) \geq 0, \forall y \in C
$$

The set of solutions to (1.1) is denoted by $\Omega$, i.e.,

$$
\Omega=\{u \in C: \Theta(u, y)+\langle A u, y-u\rangle+\psi(y)-\psi(u) \geq 0, \quad \forall y \in C\} .
$$

\section{Special examples:}

(I) If $A \equiv 0$, the problem (1.1) is equivalent to finding $u \in C$ such that

$$
\Theta(u, y)+\psi(y)-\psi(u) \geq 0, \quad \forall y \in C .
$$

which is called the mixed equilibrium problem (MEP) [1].

(II) If $\Theta \equiv 0$, the problem (1.1) is equivalent to finding $u \in C$ such that

$$
\langle A u, y-u\rangle+\psi(y)-\psi(u) \geq 0, \forall y \in C .
$$

which is called the mixed variational inequality of Browder type (VI) [2].

A Banach space $X$ is said to be strictly convex, if $\frac{\|x+y\|}{2}<1$ for all $x, y \in U=\{z \in X$ : $\|z\|=1\}$ with $x \neq y$. $X$ is said to be uniformly convex if, for each $\epsilon \in(0,2]$, there exists $\delta>0$ such that $\frac{\|x+y\|}{2}<1-\delta$ for all $x, y \in U$ with $\|x-y\| \geq \epsilon$. X is said to be smooth if the limit

$$
\lim _{t \rightarrow 0} \frac{\|x+t y\|-\|x\|}{t}
$$

exists for all $x, y \in U . X$ is said to be uniformly smooth if the above limit is attained uniformly in $x, y \in U$.

Remark 1.1 The following basic properties of a Banach space $X$ can be found in Cioranescu [1].

(i) If $X$ is uniformly smooth, then $X$ is reflexive and the normalized duality mapping $J$ is uniformly continuous on each bounded subset of $X$;

(ii) If $X$ is a reflexive and strictly convex Banach space, then $J^{-1}$ is norm-weakcontinuous;

(iii) If $X$ is a smooth, strictly convex, and reflexive Banach space, then $J$ is singlevalued, one-to-one and onto;

(iv) A Banach space $X$ is uniformly smooth if and only if $X^{*}$ is uniformly convex;

(v) Each uniformly convex Banach space $X$ has the Kadec-Klee property, i.e., for any sequence $\left\{x_{n}\right\} \subset X$, if $x_{n} \rightarrow x \in X$ and $\left\|x_{n}\right\| \rightarrow\|x\|$, then $x_{n} \rightarrow x$.

Let $X$ be a smooth Banach space. In the sequel, we use $\phi: X \times X \rightarrow \mathscr{R}^{+}$to denote the Lyapunov functional which is defined by

$$
\phi(x, y)=\|x\|^{2}-2\langle x, J y\rangle+\|y\|^{2}, \quad \forall x, y \in X .
$$

It is obvious from the definition of $\varphi$ that

$$
(\|x\|-\|y\|)^{2} \leq \phi(x, y) \leq(\|x\|+\|y\|)^{2}, \quad \forall x, y \in X
$$

and

$$
\phi\left(x, J^{-1}(\lambda J y+(1-\lambda) J z)\right) \leq \lambda \phi(x, y)+(1-\lambda) \phi(x, z)
$$


for all $\lambda \in[0,1]$ and $x, y, z \in X$. If $X$ is a smooth, strictly convex, and reflexive Banach space, following Alber [2], the generalized projection $\Pi_{C}: X \rightarrow C$ is defined by

$$
\Pi_{C}(x)=\arg \inf _{y \in C} \phi(y, x), \quad \forall x \in X .
$$

Lemma 1.2 [2] Let $X$ be a smooth, strictly convex, and reflexive Banach space and $C$ be a nonempty closed convex subset of $X$. Then the following conclusions hold:

(a) $\varphi\left(x, \Pi_{C} y\right)+\varphi\left(\Pi_{C} y, y\right) \leq \varphi(x, y)$ for all $x \in C$ and $y \in X$;

(b) If $x \in X$ and $z \in C$, then

$$
z=\Pi_{C} x \Leftrightarrow\langle z-y, J x-J z\rangle \geq 0, \quad \forall y \in C ;
$$

(c) For $x, y \in X, \varphi(x, y)=0$ if and only if $x=y$.

In the sequel, we denote by $2^{C}$ the family of all nonempty subsets of $C$.

Definition 1.3 Let $T: C \rightarrow 2^{C}$ be a multi-valued mapping.

(1) A point $p \in C$ is said to be an asymptotic fixed point of $T$, if there exists a sequence $\left\{x_{n}\right\}$ in $C$ such that $\left\{x_{n}\right\}$ converges weakly to $p$ and

$$
\lim _{n \rightarrow \infty} d\left(x_{n}, T\left(x_{n}\right)\right):=\lim _{n \rightarrow \infty} \inf _{\gamma \in T\left(x_{n}\right)}\left\|x_{n}-y\right\|=0 .
$$

In the sequel we use $\hat{F}(T)$ to denote the set of all asymptotic fixed points of $T$;

(2) A multi-valued mapping $T: C \rightarrow 2^{C}$ is said to be relatively nonexpansive [3], if

(a) $F(T) \neq \varnothing$;

(b) $\varphi(p, w) \leq \varphi(p, x), \forall x \in C, w \in T x, p \in F(T)$

(c) $\hat{F}(T)=F(T)$.

Definition 1.4 (1) A multi-valued mapping $T: C \rightarrow 2^{C}$ is said to be quasi- $\varphi$-nonexpansive, if $F(T) \neq \varnothing$ and

$$
\phi(p, w) \leq \phi(p, x), \quad \forall x \in C, w \in T x, p \in F(T) .
$$

(2) A multi-valued mapping $T: C \rightarrow 2^{C}$ is said to be quasi- $\varphi$-asymptotically nonexpansive if $F(T) \neq \varnothing$ and there exists a real sequence $\left\{k_{n}\right\} \subset[1, \infty)$ with $k_{n} \rightarrow 1$ such that

$$
\phi\left(p, w_{n}\right) \leq k_{n} \phi(p, x), \quad \forall n \geq 1, x \in C, w_{n} \in T^{n} x, p \in F(T) .
$$

(3) A multi-valued mapping $T: C \rightarrow 2^{C}$ is said to be $\left(\left\{v_{n}\right\},\left\{\mu_{n}\right\}, \zeta\right)$-total quasi- $\varphi$ asymptotically nonexpansive, if $F(T) \neq \varnothing$ and there exist nonnegative real sequences $\left\{v_{n}\right\},\left\{\mu_{n}\right\}$ with $v_{n} \rightarrow 0, \mu_{n} \rightarrow 0$ (as $n \rightarrow \infty$ ) and a strictly increasing continuous function $\zeta: \mathscr{R}^{+} \rightarrow \mathscr{R}^{+}$with $\zeta(0)=0$ such that for all $x \in C, p \in F(T)$

$$
\phi\left(p, w_{n}\right) \leq \phi(p, x)+v_{n} \zeta(\phi(p, x))+\mu_{n}, \quad \forall n \geq 1, w_{n} \in T^{n} x .
$$

(4) A total quasi- $\varphi$-asymptotically nonexpansive multi-valued mapping $T: C \rightarrow 2^{C}$ is said to be uniformly L-Lipschitz continuous if there exists a constant $L>0$ such that

$$
\left\|w_{n}-s_{n}\right\| \leq L|| x-y \|, \quad \forall x, y \in C, w_{n} \in T^{n} x, s_{n} \in T^{n} y, n \geq 1 .
$$

(5) A multi-valued mapping $T: C \rightarrow 2^{C}$ is said to be closed if, for any sequences $\left\{x_{n}\right\}$ and $\left\{w_{n}\right\}$ in $C$ with $w_{n} \in T\left(x_{n}\right)$, if $x_{n} \rightarrow x$ and $w_{n} \rightarrow y$, then $y \in T x$. 
(6) A countable family of multi-valued mappings $\left\{T_{i}\right\}_{i=1}^{\infty}: C \rightarrow 2^{C}$ is said to be uniformly $\left(\left\{v_{n}\right\},\left\{\mu_{n}\right\}, \zeta\right)$-total quasi- $\varphi$-asymptotically nonexpansive, if $\mathscr{F}:=\bigcap_{i=1}^{\infty} F\left(T_{i}\right) \neq \varnothing$ and there exist nonnegative real sequences $\left(\left\{v_{n}\right\},\left\{\mu_{n}\right\}\right.$ with $v_{n} \rightarrow 0, \mu_{n} \rightarrow 0$ and a strictly increasing continuous function $\zeta: \mathscr{R}^{+} \rightarrow \mathscr{R}^{+}$with $\zeta(0)=0$ such that for all $x$ $\in C, p \in \mathscr{F}$

$$
\phi\left(p, w_{n, i}\right) \leq \phi(p, x)+v_{n} \zeta(\phi(p, x))+\mu_{n}, \quad \forall n \geq 1, w_{n, i} \in T_{i}^{n} x, i=1,2, \ldots
$$

Remark 1.5 From the definitions, it is easy to know that

(1) Every quasi- $\varphi$-asymptotically nonexpansive multi-valued mapping must be a total quasi- $\varphi$-asymptotically nonexpansive multi-valued mapping. In fact, taking $\zeta(t)=t, t \geq 0$, $k_{n}=v_{n}+1$ and $\mu_{n}=0$, then (1.6) can be rewritten as

$$
\phi\left(p, w_{n}\right) \leq \phi(p, x)+v_{n} \zeta(\phi(p, x))+\mu_{n}, \quad \forall n \geq 1, x \in C, w_{n} \in T^{n} x, p \in F(T),
$$

where $v_{n} \rightarrow 0($ as $n \rightarrow \infty)$.

(2) The class of quasi- $\varphi$-asymptotically nonexpansive multi-valued mappings contains properly the class of quasi- $\varphi$-nonexpansive multi-valued mappings as a subclass, but the converse is not true.

(3) The class of quasi- $\varphi$-nonexpansive multi-valued mappings contains properly the class of relatively nonexpansive multi-valued mappings as a subclass, but the converse is not true.

Example 1.6 Now we give some examples of single-valued and multi-valued total quasi- $\varphi$-asymptotically nonexpansive mappings.

(1) Single-valued total quasi- $\varphi$-asymptotically nonexpansive mapping.

Let $C$ be a unit ball in a real Hilbert space $l^{2}$ and let $T: C \rightarrow C$ be a mapping defined by

$$
T:\left(x_{1}, x_{2}, \ldots,\right) \rightarrow\left(0, x_{1}^{2}, a_{2} x_{2}, a_{3} x_{3}, \ldots\right),\left(x_{1}, x_{2}, \ldots,\right) \in l^{2},
$$

where $\left\{a_{i}\right\}$ is a sequence in $(0,1)$ such that $\prod_{i=2}^{\infty} a_{i}=\frac{1}{2}$. It is proved in [4] that $T$ is total quasi- $\varphi$-asymptotically nonexpansive.

(2) Multi-valued total quasi- $\varphi$-asymptotically nonexpansive mappings.

Let $I=0[1], X=C(I)$ (the Banach space of continuous functions defined on $I$ with the uniform convergence norm $\left.\|f\|_{C}=\sup _{t_{L} I}|f(t)|\right), D=\{f \in X: f(x) \geq 0, \forall x \in I\}$ and $a$, $b$ be two constants in $(0,1)$ with $a<b$. Let $T: D \rightarrow 2^{D}$ be a multi-valued mapping defined by

$$
T(f)=\left\{\begin{array}{lc}
\{g \in D: a \leq f(x)-g(x) \leq b, \forall x \in I\}, & \text { if } f(x)>1, \forall x \in I \\
\{0\}, & \text { otherwise. }
\end{array}\right.
$$

It is easy to see that $F(T)=\{0\}$, therefore $F(T)$ is nonempty.

Next, we prove that $T: D \rightarrow 2^{D}$ is a closed total quasi- $\varphi$-asymptotically nonexpansive multi-valued mapping. In fact, for any given $f \in D$ :

(I) if $f(x)>1, \forall x \in I$, then for any $g \in T(f)$, we have $a \leq f(x)-g(x) \leq b$. Hence for any $p \in F(T)=\{0\}$ we have

$$
\phi(p, g)=\phi(0, g)=\|g\|_{C}^{2} \leq\|f\|_{C}^{2}=\phi(0, f)=\phi(p, f) .
$$


If there exists some point $x_{0} \in I$ such that $0 \leq f\left(x_{0}\right) \leq 1$, then from the definition of mapping $T$, we have $T(f)=\{0\}$. Hence for any $p \in F(T)$ and $g \in T(f)=\{0\}$, we have

$$
\phi(p, g)=\phi(0,0)=0 \leq\|f\|_{C}^{2}=\phi(0, f)=\phi(p, f) .
$$

Summing up the above arguments we have that for any given $f \in D$

$$
\phi(p, g) \leq \phi(p, f), \quad \forall p \in F(T), g \in T(f),
$$

(II) For any $g \in T^{2}(f)=T(T(f))=\bigcup_{g_{1} \in T(f)} T\left(g_{1}\right)$, there exists some $g_{1}^{*} \in T(f)$ such that $g \in T\left(g_{1}^{*}\right)$.

(1) If $g_{1}^{*}>1, \forall x \in I$, then we have $a \leq g_{1}^{*}-g<b$. By (1.13), for any $p \in F(T)=\{0\}$, we have

$$
\phi(p, g)=\phi(0, g)=\|g\|_{C}^{2} \leq\left\|g_{1}^{*}\right\|_{C}^{2}=\phi\left(0, g_{1}^{*}\right)=\phi\left(p, g_{1}^{*}\right) \leq \phi(p, f) .
$$

(2) If there exists $x_{1} \in I$ such that $0 \leq g_{1}^{*}\left(x_{1}\right) \leq 1$, then by the definition of $T$, we have $T g_{1}^{*}=\{0\}$. Since $g \in T g_{1}^{*}=\{0\}$, and so $g=0$. Hence for any $p \in F(T)$, by (1.13) we have

$$
\phi(p, g)=\phi(0,0)=0 \leq\left\|g_{1}^{*}\right\|^{2}=\phi\left(0, g_{1}^{*}\right)=\phi\left(p, g_{1}^{*}\right) \leq \phi(p, f) .
$$

From (1) and (2) we have that for any given $f \in D$

$$
\phi(p, g) \leq \phi(p, f), \quad \forall p \in F(T), g \in T^{2}(f),
$$

By induction, we can prove that for any given $f \in D, g \in T^{n}(f), n \geq 1, p \in F(T)$,

$$
\phi(p, g) \leq \phi(p, f) .
$$

Letting $\left\{\mu_{n}\right\}$ and $\left\{v_{n}\right\}$ be two any nonnegative sequences with $\mu_{n} \rightarrow 0$ and $v_{n} \rightarrow 0$ and $\zeta(t)=t, t \geq 0$, then (1.15) can be rewritten as

$$
\phi(p, g) \leq \phi(p, f)+v_{n} \zeta(\phi(p, f))+\mu_{n}
$$

for any $f \in D, g \in T^{n}(f), n \geq 1, p \in F(T)$. This shows that $T: C \rightarrow 2^{C}$ is a total quasi$\varphi$-asymptotically nonexpansive multi-valued mapping.

Next, we prove that $T$ is a closed mapping. In fact, let $\left\{f_{n}\right\}$ and $\left\{g_{n}\right\}$ be two sequences in $D$ with $g_{n} \in T\left(f_{n}\right)$ such that $\left\|f_{n}-f\right\|_{C} \rightarrow 0,\left\|g_{n}-g\right\|_{C} \rightarrow 0$ as $n \rightarrow \infty$.

(1) If $f(x)>1, \forall x \in I$, since $\left\{f_{n}\right\}$ converges uniformly to $f$, then there exists $n_{0} \geq 1$ such that $f_{n}(x)>1, \forall x \in I, \forall n \geq n_{0}$. By the definition of $T$, we have

$$
a \leq f_{n}(x)-g_{n}(x) \leq b, \forall n \geq 1 \text { and } x \in I .
$$

Letting $n \rightarrow \infty$ in (1.16), we have

$$
a \leq f(x)-g(x) \leq b, \quad \forall n \geq 1 .
$$

This implies that $g \in T(f)$.

(2) If there exists some point $x_{2} \in I$ such that $0 \leq f\left(x_{2}\right) \leq 1$, then $T(f)=\{0\}$. Since $\left\{f_{n}\right\}$ converges uniformly to $f$, then there exists a positive integer $n_{2}$ such that $0 \leq f_{n}$ $\left(x_{2}\right) \leq 1, \forall n \geq n_{2}$. By the definition of $T$, this implies that $T\left(f_{n}\right)=0, \forall n \geq n_{2}$. Since $g_{n}$ $\in T\left(f_{n}\right)$, this implies that $g_{n}=0, \forall n \geq n_{2}$. Since $g_{n} \rightarrow g, g=0$. Therefore $g \in T(f)$. 
These show that $T$ is a closed mapping.

Concerning the weak and strong convergence of iterative sequences to approximate a common element of the set of solutions for a generalized MEP, the set of solutions for variational inequality problems, and the set of common fixed points for single-valued relatively non-expansive mappings, single-valued quasi- $\varphi$-nonexpansive mappings, single-valued quasi- $\varphi$-asymptotically nonexpansive mappings and single-valued total quasi$\varphi$-asymptotically non-expansive mappings have been studied by many authors in the setting of Hilbert or Banach spaces (see, for example, [4-21] and the references therein). Very recently, in 2011, Homaeipour and Razani [3] introduced the concept of multivalued relatively nonexpansive mappings and proved some weak and strong convergence theorems to approximation a fixed point for a single relatively nonexpansive multivalued mapping in a uniformly convex and uniformly smooth Banach space $X$ which improve and extend the corresponding results of Matsushita and Takahashi [5].

Motivated and inspired by the researches going on in this direction, the purpose of this article is first to introduce the concept of total quasi- $\varphi$-asymptotically nonexpansive multi-valued mapping which contains multi-valued relatively nonexpansive mappings and many other kinds of mappings as its special cases, and then by using the hybrid shirking iterative algorithm for finding a common element of the set of solutions for a generalized MEP, the set of solutions for variational inequality problems, and the set of common fixed points for a countable family of multi-valued total quasi- $\varphi$-asymptotically nonexpansive mappings in a real uniformly smooth and strictly convex Banach space with Kadec-Klee property. The results presented in the article not only generalize the corresponding results of [4-21] from single-valued mappings to multi-valued mappings, but also improve and extend the main results of Homaeipour and Razani [3]. The method given in this article is quite different from that one adopted in [3].

\section{Preliminaries}

In order to prove our main results, the following conclusions and notations will be needed.

Lemma 2.1 [8] Let $X$ be a real uniformly smooth and strictly convex Banach space with Kadec-Klee property, and $C$ be a nonempty closed convex set of $X$. Let $\left\{x_{n}\right\}$ and $\left\{y_{n}\right\}$ be two sequences in $C$ such that $x_{n} \rightarrow p$ and $\varphi\left(x_{n}, y_{n}\right) \rightarrow 0$, where $\varphi$ is the function defined by (1.1), then $y_{n} \rightarrow p$.

Lemma 2.2 Let $X$ and $C$ be as in Lemma 2.1. Let $T: C \rightarrow 2^{C}$ be a closed and $\left(\left\{v_{n}\right\}\right.$, $\left.\left\{\mu_{n}\right\}, \zeta\right)$-total quasi- $\varphi$-asymptotically nonexpansive multi-valued mapping. If $\mu_{1}=0$, then the fixed point set $F(T)$ of $T$ is a closed and convex subset of $C$.

Proof Let $\left\{x_{n}\right\}$ be a sequence in $F(T)$ with $x_{n} \rightarrow p($ as $n \rightarrow \infty)$, we prove that $p \in F$ (T). In fact, by the assumption that $T$ is a $\left(\left\{v_{n}\right\},\left\{\mu_{n}\right\}, \zeta\right)$-total quasi- $\varphi$-asymptotically nonexpansive multi-valued mapping with $\mu_{1}=0$, hence we have

$$
\phi\left(x_{n}, u\right) \leq \phi\left(x_{n}, p\right)+v_{1} \zeta\left(\phi\left(x_{n}, p\right)\right), \quad \forall u \in T p,
$$

and

$$
\begin{aligned}
\phi(p, u) & =\lim _{n \rightarrow \infty} \phi\left(x_{n}, u\right) \\
& \leq \lim _{n \rightarrow \infty}\left(\phi\left(x_{n}, p\right)+v_{1} \zeta\left(\phi\left(x_{n}, p\right)\right)\right)=0, \forall u \in T p .
\end{aligned}
$$


By Lemma 1.2(c), $p=u$. Hence, $p \in T p$. This implies that $F(T)$ is a closed set in $C$.

Next, we prove that $F(T)$ is convex. For any $x, y \in F(T), t \in(0,1)$, putting $q=t x+$ $(1-t) y$, we prove that $q \in F(T)$. Indeed, let $\left\{u_{n}\right\}$ be a sequence generated by

$$
\begin{gathered}
u_{1} \in T q, \quad u_{2} \in T u_{1} \subset T^{2} q, \quad u_{3} \in T u_{2} \subset T^{3} q_{1} \ldots \\
u_{n} \in T u_{n-1} \subset T^{n} q_{1} \ldots
\end{gathered}
$$

Therefore for each $u_{n} \in T u_{n-1} \subset T^{n} q$, we have

$$
\begin{aligned}
\phi\left(q, u_{n}\right) & =\|q\|^{2}-2\left\langle q, J u_{n}\right\rangle+\left\|u_{n}\right\|^{2} \\
& =\|q\|^{2}-2 t\left\langle x, J u_{n}\right\rangle-2(1-t)\left\langle y, J u_{n}\right\rangle+\left\|u_{n}\right\|^{2} \\
& =\|q\|^{2}+t \phi\left(x, u_{n}\right)+(1-t) \phi\left(y, u_{n}\right)-t\|x\|^{2}-(1-t)\|y\|^{2}
\end{aligned}
$$

Since

$$
\begin{aligned}
& t \phi\left(x, u_{n}\right)+(1-t) \phi\left(y, u_{n}\right) \\
\leq & t\left(\phi(x, q)+v_{n} \zeta(\phi(x, q))+\mu_{n}\right)+(1-t)\left(\phi(y, q)+v_{n} \zeta(\phi(y, q))+\mu_{n}\right) \\
= & t\left(\|x\|^{2}-2\langle x, J q\rangle+\|q\|^{2}+v_{n} \zeta(\phi(x, q))+\mu_{n}\right) \\
& +(1-t)\left(\|y\|^{2}-2\langle y, J q\rangle+\|q\|^{2}+v_{n} \zeta(\phi(y, q))+\mu_{n}\right) \\
= & t\|x\|^{2}+(1-t)\|y\|^{2}-\|q\|^{2}+t v_{n} \zeta(\phi(x, q))+(1-t) v_{n} \zeta(\phi(\gamma, q))+\mu_{n}
\end{aligned}
$$

Substituting (2.3) into (2.2) and simplifying we have

$$
\phi\left(q, u_{n}\right) \leq t v_{n} \zeta(\phi(x, q))+(1-t) v_{n} \zeta(\phi(\gamma, q))+\mu_{n} \rightarrow 0(n \rightarrow \infty) .
$$

By Lemma 2.1, we have $u_{n} \rightarrow q$ (as $\left.n \rightarrow \infty\right)$. This implies that $u_{n+1} \rightarrow q($ as $n \rightarrow \infty)$. Since $u_{n+1} \in T u_{n}$ and $T$ is closed, we have $q \in T q$, i.e., $q \in F(T)$.

This completes the proof of Lemma 2.2.

Lemma 2.3 [8] Let $X$ be a uniformly convex Banach space, $r>0$ be a positive number and $B_{r}(0)$ be a closed ball of $X$. Then for any sequence $\left\{x_{i}\right\}_{i=1}^{\omega} \subset B_{r}(0)$ (where $\omega$ is any positive integer or $+\infty$ ) and for any sequence $\left\{\lambda_{i}\right\}_{i=1}^{\omega}$ of positive numbers with $\sum_{n=1}^{\omega} \lambda_{n}=1$, there exists a continuous, strictly increasing, and convex function $g:[0$, $2 r) \rightarrow[0, \infty), g(0)=0$ such that for any positive integer $i \neq 1$, the following hold:

$$
\left\|\sum_{n=1}^{\omega} \lambda_{n} x_{n}\right\|^{2} \leq \sum_{n=1}^{\omega} \lambda_{n}\left\|x_{n}\right\|^{2}-\lambda_{1} \lambda_{i} g\left(\left\|x_{1}-x_{i}\right\|\right),
$$

and for all $x \in X$

$$
\phi\left(x, J^{-1}\left(\sum_{i=1}^{\omega} \lambda_{i} J x_{i}\right) \leq \sum_{i=1}^{\omega} \lambda_{i} \phi\left(x, x_{i}\right)-\lambda_{1} \lambda_{i} g\left(\left\|J x_{1}-J x_{i}\right\|\right) .\right.
$$

For solving the generalized MEP, let us assume that the function $\psi: C \rightarrow \mathscr{R}$ is convex and lower semi-continuous, the nonlinear mapping $A: C \rightarrow X^{*}$ is continuous and monotone, and the bifunction $\Theta: C \times C \rightarrow \mathscr{R}$ satisfies the following conditions:

$\left(A_{1}\right) \Theta(x, x)=0, \forall x \in C$.

$\left(A_{2}\right) \Theta$ is monotone, i.e., $\Theta(x, y)+\Theta(y, x) \leq 0, \forall x, y \in C$.

$\left(A_{3}\right) \lim \sup _{t \downarrow} \Theta(x+t(z-x), y) \leq \Theta(x, y), \forall x, y, z \in C$.

$\left(A_{4}\right)$ The function $y \mapsto \Theta(x, y)$ is convex and lower semicontinuous. 
Lemma 2.4 Let $X$ be a smooth, strictly convex, and reflexive Banach space, and $C$ be a nonempty closed convex subset of $X$. Let $\Theta: C \times C \rightarrow \mathscr{R}$ be a bifunction satisfying the conditions $\left(A_{1}\right)-\left(A_{4}\right)$. Let $r>0$ and $x \in X$. Then, the following hold:

(i) [12] There exists $z \in C$ such that

$$
\Theta(z, y)+\frac{1}{r}\langle y-z, J z-J x\rangle \geq 0, \quad \forall y \in C .
$$

(ii) [13] Define a mapping $T_{r}: X \rightarrow C$ by

$$
T_{r} x=\left\{z \in C: \Theta(z, y)+\frac{1}{r}\langle y-z, J z-J x\rangle \geq 0, \forall y \in C\right\}, \quad x \in X .
$$

Then, the following conclusions hold:

(a) $T_{r}$ is single-valued;

(b) $T_{r}$ is a firmly nonexpansive-type mapping, i.e., $\forall z, y \in X$,

$$
\left\langle T_{r}(z)-T_{r}(y), J T_{r}(z)-J T_{r}(y)\right\rangle \leq\left\langle T_{r}(z)-T_{r}(y), J z-J y\right\rangle ;
$$

(c) $F\left(T_{r}\right)=E P(\Theta)=F\left(T_{r}\right)$;

(d) $E P(\Theta)$ is closed and convex;

(e) $\varphi\left(q, T_{r}(x)\right)+\varphi\left(T_{r}(x), x\right) \leq \varphi(q, x), \forall q \in F\left(T_{r}\right)$.

Lemma 2.5 [18] Let $X$ be a smooth, strictly convex, and reflexive Banach space, and $C$ be a nonempty closed convex subset of $X$. Let $A: C \rightarrow X^{*}$ be a continuous and monotone mapping, $\psi: C \rightarrow \mathscr{R}$ be a lower semi-continuous and convex function, and $\Theta: C \times C \rightarrow \mathscr{R}$ be a bifunction satisfying the conditions $\left(A_{1}\right)-\left(A_{4}\right)$. Let $r>0$ be any given number and $x \in X$ be any given point. Then, the following conclusions hold:

(i) There exists $u \in C$ such that $\forall y \in C$

$$
\Theta(u, y)+\langle A u, y-u\rangle+\psi(y)-\psi(u)+\frac{1}{r}\langle y-u, J u-J x\rangle \geq 0 .
$$

(ii) If we define a mapping $K_{r}: C \rightarrow C$ by

$$
\begin{gathered}
K_{r}(x)=\{u \in C: \Theta(u, y)+\langle A u, y-u\rangle+\psi(y)-\psi(u) \\
\left.+\frac{1}{r}\langle y-u, J u-J x\rangle \geq 0, \forall y \in C\right\}, \quad x \in C,
\end{gathered}
$$

then, the mapping $K_{r}$ has the following properties:

(a) $K_{r}$ is single-valued;

(b) $K_{r}$ is a firmly nonexpansive-type mapping, i.e., $\forall z, y \in X$

$$
\left\langle K_{r}(z)-K_{r}(\gamma), J K_{r}(z)-J K_{r}(y)\right\rangle \leq\left\langle K_{r}(z)-K_{r}(\gamma), J z-J y\right\rangle ;
$$

(c) $F\left(K_{r}\right)=\Omega=F\left(K_{r}\right)$;

(d) $\Omega$ is a closed convex set of $C$;

(e) $\varphi\left(p, K_{r}(z)\right)+\varphi\left(K_{r}(z), z\right) \leq \varphi(p, z), \forall p \in F\left(K_{r}\right), z \in X$.

Remark 2.6 It follows from Lemma 2.4 that the mapping $K_{r}: C \rightarrow C$ defined by (2.6) is a relatively nonexpansive mapping. Thus, it is quasi- $\varphi$-nonexpansive. 


\section{Main results}

In this section, we shall use the hybrid iterative algorithm to find a common element of the set of solutions of a generalized MEP, the set of solutions for variational inequality problems, and the set of fixed points of a infinite family of total quasi- $\varphi$ asymptotically nonexpansive multi-valued mappings. For the purpose we give the following hypotheses:

(H1) $X$ is a uniformly smooth and strictly convex Banach space with Kadec-Klee property and $C$ is a nonempty closed convex subset of $X$;

$(\mathrm{H} 2) \Theta: C \times C \rightarrow \mathscr{R}$ is a bifunction satisfying the conditions $\left(A_{1}\right)-\left(A_{4}\right), A: C \rightarrow X^{*}$ is a continuous and monotone mapping, and $\psi: C \rightarrow \mathscr{R}$ is a lower semi-continuous and convex function.

(H3) $\left\{T_{i}\right\}_{i=1}^{\infty}: C \rightarrow 2^{C}$ is a countable family of closed and uniformly $\left(\left\{v_{n}\right\},\left\{\mu_{n}\right\}, \zeta\right)$ total quasi- $\varphi$-asymptotically nonexpansive multi-valued mappings and for each $i=1,2$, $\ldots, T_{i}$ is uniformly $L_{i}$-Lipschitzian with $\mu_{1}=0$.

We have the following

Theorem 3.1. Let $X, C, \Theta, A, \psi,\left\{T_{i}\right\}_{i=1}^{\infty}$ satisfy the above conditions (H1)-(H3). Let $\left\{x_{n}\right\}$ be the sequence generated by

$$
\left\{\begin{array}{l}
x_{0} \in C \text { chosen arbitrary, } \quad C_{0}=C, \\
y_{n}=J^{-1}\left(\alpha_{n} J x_{n}+\left(1-\alpha_{n}\right) J z_{n}\right), \quad \forall n \geq 1, \\
z_{n}=J^{-1}\left(\beta_{n, 0} J x_{n}+\sum_{i=1}^{\infty} \beta_{n, i} J w_{n, i}\right), \quad\left(w_{n, i} \in T_{i}^{n} x_{n}, i \geq 1\right), \forall n \geq 1, \\
u_{n} \in C \text { such that } \forall y \in C, \forall n \geq 1, \\
\Theta\left(u_{n}, \gamma\right)+\left\langle A u_{n}, y-u_{n}\right\rangle+\psi(y)-\psi\left(u_{n}\right)+\frac{1}{r_{n}}\left\langle y-u_{n} J u_{n}-J y_{n}\right\rangle \geq 0, \\
C_{n+1}=\left\{v \in C_{n}: \phi\left(v, u_{n}\right) \leq \phi\left(v, x_{n}\right)+\xi_{n}\right\}, \quad \forall n \geq 0 \\
x_{n+1}=\Pi_{C_{n+1}} x_{0}, \forall n \geq 0
\end{array}\right.
$$

where $\prod_{C_{n+1}}$ is the generalized projection of $X$ onto $C_{n+1}, \mathscr{F}:=\bigcap_{i=1}^{\infty} F\left(T_{i}\right)$, $\xi_{n}=v_{n} \sup _{p \in \mathscr{F}} \zeta\left(\phi\left(p, x_{n}\right)\right)+\mu_{n},\left\{\alpha_{n}\right\}$ and $\left\{\beta_{n, 0}, \beta_{n, i}\right\}$ are sequences in $0[1]$ satisfying the following conditions:

(i) for each $n \geq 0, \sum_{i=0}^{\infty} \beta_{n, i}=1$;

(ii) $\lim \inf _{n \rightarrow \infty} \beta_{n, 0}, \beta_{n i}>0$ for any $i \geq 1$;

(iii) $0 \leq \alpha_{n} \leq \alpha<1$ for some $\alpha \in(0,1)$.

If $\mathscr{G}:=\mathscr{F} \cap \Omega=\cap_{i=1}^{\infty} F\left(T_{i}\right) \cap \Omega$ is nonempty and $\mathscr{F}$ is a bounded subset of $C$, then the sequence $\left\{x_{n}\right\}$ converges strongly to $\prod_{G} x_{0}$.

Proof. First, we define two functions $H: C \times C \rightarrow \mathscr{R}$ and $K_{r}: C \rightarrow C$ by

$$
\begin{aligned}
& H(x, y)=\Theta(x, y)+\langle A x, y-x\rangle+\psi(y)-\psi(x), \quad \forall x, y \in C, \\
& K_{r}(x)=\left\langle u \in C: H(u, y)+\frac{1}{r}\langle y-u, J u-J x\rangle \geq 0, \quad \forall y \in C\right\}, \quad x \in C .
\end{aligned}
$$

By Lemma 2.5, we know that the function $H$ satisfies the conditions $\left(A_{1}\right)-\left(A_{4}\right)$ and $K_{r}$ has the property (a)-(e). Therefore, (3.1) can be rewritten as 
Now we divide the proof of Theorem 3.1 into six steps.

(I) $\mathscr{F}$ and $C_{n}$ are closed and convex for each $n \geq 0$.

In fact, it follows from Lemma 2.2 that $F\left(T_{i}\right), i \geq 1$ is closed and convex subsets of $C$. Therefore $\mathscr{F}$ is a closed and convex subsets in $C$.

Again by the assumption, $C_{0}=C$ is closed and convex. Suppose that $C_{n}$ is closed and convex for some $n \geq 1$. Since the condition $\varphi\left(v, y_{n}\right) \leq \varphi\left(v, x_{n}\right)+\xi_{n}$ is equivalent to

$$
2\left\langle v, J x_{n}-J y_{n}\right\rangle \leq\left\|x_{n}\right\|^{2}-\left\|y_{n}\right\|^{2}+\xi_{n}, \quad n=1,2, \ldots,
$$

hence the set

$$
C_{n+1}=\left\{v \in C_{n}: 2\left\langle v, J x_{n}-J y_{n}\right\rangle \leq\left\|x_{n}\right\|^{2}-\left\|y_{n}\right\|^{2}+\xi_{n}\right\}
$$

is closed and convex. Therefore $C_{n}$ is closed and convex for each $n \geq 0$.

(II) $\left\{x_{n}\right\}$ is bounded and $\left\{\varphi\left(x_{n}, x_{0}\right)\right\}$ is a convergent sequence.

Indeed, it follows from (3.1) and Lemma 1.2(a) that for all $n \geq 0, u \in F(T)$

$$
\phi\left(x_{n}, x_{0}\right)=\phi\left(\Pi_{C_{n}} x_{0}, x_{0}\right) \leq \phi\left(u, x_{0}\right)-\phi\left(u, \Pi_{C_{n}} x_{0}\right) \leq \phi\left(u, x_{0}\right) .
$$

This implies that $\left\{\varphi\left(x_{n}, x_{0}\right)\right\}$ is bounded. By virtue of (1.6), we know that $\left\{x_{n}\right\}$ is bounded.

In view of structure of $\left\{C_{n}\right\}$, we have $C_{n+1} \subset C_{n}, x_{n}=\prod_{C_{n}} x_{0}$ and $x_{n+1}=\prod_{C_{n+1}} x_{0}$. This implies that $x_{n+1} \in C_{n}$ and

$$
\phi\left(x_{n}, x_{0}\right) \leq \phi\left(x_{n+1}, x_{0}\right), \quad \forall n \geq 0 .
$$

Therefore $\left\{\varphi\left(x_{n}, x_{0}\right)\right\}$ is a convergent sequence.

(III) $\mathscr{G}:=\mathscr{F} \cap \Omega \subset C_{n}$ for all $n \geq 0$.

Indeed, it is obvious that $\mathscr{G} \subset C_{0}=C$. Suppose that $\mathscr{G} \subset C_{n}$ for some $n \in \mathscr{N}$. Since $u_{n}=K_{r_{n}} y_{n}$, by Lemma 2.5 and Remark 2.6, $K_{r_{n}}$ is quasi- $\varphi$-nonexpansive. Hence, for any given $u \in \mathscr{G} \subset C_{n}$ and $n \geq 1$, it follows from (1.7) that

$$
\begin{aligned}
\phi\left(u, u_{n}\right) & =\phi\left(u, K_{r_{n}} y_{n}\right) \leq \phi\left(u, y_{n}\right) \\
& =\phi\left(u, J^{-1}\left(\alpha_{n} J x_{n}+\left(1-\alpha_{n}\right) J z_{n}\right)\right) \\
& \leq \alpha_{n} \phi\left(u, x_{n}\right)+\left(1-\alpha_{n}\right) \phi\left(u, z_{n}\right) .
\end{aligned}
$$

Furthermore, it follows from Lemma 2.3 that for any $u \in G \subset C_{n}, w_{n, i} \in T_{i}^{n} x_{n}$ and $i$ $\geq 1$ we have 


$$
\begin{aligned}
\phi\left(u, z_{n}\right)= & \phi\left(u, J^{-1}\left(\beta_{n, 0} J x_{n}+\sum_{i=1}^{\infty} \beta_{n, i} J w_{n, i}\right)\right) \\
\leq & \beta_{n, 0} \phi\left(u, x_{n}\right)+\sum_{i=1}^{\infty} \beta_{n, i} \phi\left(u, w_{n, i}\right)-\beta_{n, 0} \beta_{n, l} g\left(\left\|J x_{n}-J w_{n, l}\right\|\right) \\
\leq & \beta_{n, 0} \phi\left(u, x_{n}\right)+\sum_{i=1}^{\infty} \beta_{n, i}\left(\phi\left(u, w_{n, i}\right)+v_{n} \zeta\left(\phi\left(u, w_{n, i}\right)\right)+\mu_{n}\right) \\
& -\beta_{n, 0} \beta_{n, l} g\left(\left\|J x_{n}-J w_{n, l}\right\|\right) \\
\leq & \phi\left(u, x_{n}\right)+v_{n} \sup _{p \in \mathscr{F}} \zeta\left(\phi\left(p, x_{n}\right)\right)+\mu_{n}-\beta_{n, 0} \beta_{n, l} g\left(\left\|J x_{n}-J w_{n, l}\right\|\right) \\
= & \phi\left(u, x_{n}\right)+\xi_{n}-\beta_{n, 0} \beta_{n, l} g\left(\left\|J x_{n}-J w_{n, l}\right\|\right),
\end{aligned}
$$

where $\xi_{n}=v_{n} \sup _{p \in \mathscr{F}} \zeta\left(\phi\left(p, x_{n}\right)\right)$. Substituting (3.4) into (3.3) and simplifying, $\forall u \in \mathscr{G}$ we have

$$
\begin{aligned}
\phi\left(u, u_{n}\right) & \leq \phi\left(u, y_{n}\right) \\
& \leq \phi\left(u, x_{n}\right)+\left(1-\alpha_{n}\right) \xi_{n}-\left(1-\alpha_{n}\right) \beta_{n, 0} \beta_{n, l}\left(\left\|J x_{n}-J w_{n, l}\right\|\right) \\
& \leq \phi\left(u, x_{n}\right)+\xi_{n}-\left(1-\alpha_{n}\right) \beta_{n, 0} \beta_{n, l} g\left(\| J x_{n}-J w_{n, l}||\right) \\
& \leq \phi\left(u, x_{n}\right)+\xi_{n},
\end{aligned}
$$

i.e., $u \in C_{n+1}$ and so $\mathscr{G} \subset C_{n+1}$ for all $n \geq 0$.

By the way, in view of the assumption on $\left\{v_{n}\right\},\left\{\mu_{n}\right\}$ we have

$$
\xi_{n}=v_{n} \sup _{p \in \mathscr{F}} \zeta\left(\phi\left(p, x_{n}\right)\right)+\mu_{n} \rightarrow 0(n \rightarrow \infty) .
$$

(IV) $\left\{x_{n}\right\}$ converges strongly to some point $p^{*} \in C$.

In fact, since $\left\{x_{n}\right\}$ is bounded and $X$ is reflexive, there exists a subsequence $\left\{x_{n_{i}}\right\} \subset\left\{x_{n}\right\}$ such that $x_{n_{i}} \rightarrow p^{*}$ (some point in $C$ ). Since $C_{n}$ is closed and convex and $C_{n+1} \subset C_{n}$, this implies that $C_{n}$ is weakly closed and $p^{*} \in C_{n}$ for each $n \geq 0$. In view of $x_{n_{i}}=\Pi_{C_{n_{i}}} x_{0}$, we have

$$
\phi\left(x_{n_{i}}, x_{0}\right) \leq \phi\left(p^{*}, x_{0}\right), \quad \forall n_{i} \geq 0
$$

Since the norm $\|\cdot\|$ is weakly lower semi-continuous, we have

$$
\begin{aligned}
\liminf _{n_{i} \rightarrow \infty} \phi\left(x_{n_{i}}, x_{0}\right) & =\liminf _{n_{i} \rightarrow \infty}\left(\left\|x_{n_{i}}\right\|^{2}-2\left\langle x_{n i}, J x_{0}\right\rangle+\left\|x_{0}\right\|^{2}\right) \\
& \geq\left\|p^{*}\right\|^{2}-2\left\langle p^{*}, J x_{0}\right\rangle+\left\|x_{0}\right\|^{2}=\phi\left(p^{*}, x_{0}\right),
\end{aligned}
$$

and so

$$
\phi\left(p^{*}, x_{0}\right) \leq \liminf _{n_{i} \rightarrow \infty} \phi\left(x_{n_{i}}, x_{0}\right) \leq \limsup _{n_{i} \rightarrow \infty} \phi\left(x_{n_{i}}, x_{0}\right) \leq \phi\left(p^{*}, x_{0}\right)
$$

This implies that $\lim _{n_{i} \rightarrow \infty} \phi\left(x_{n_{i}}, x_{0}\right)=\phi\left(p^{*}, x_{0}\right)$, and so $\left\|x_{n_{i}}\right\| \rightarrow\left\|p^{*}\right\|$. Since $x_{n_{i}} \rightarrow p^{*}$, by virtue of Kadec-Klee property of $X$, we obtain that

$$
\lim _{n_{i} \rightarrow \infty} x_{n_{i}}=p^{*}
$$

Since $\left\{\varphi\left(x_{n}, x_{0}\right)\right\}$ is convergent, this together with $\lim _{n_{i} \rightarrow \infty} \phi\left(x_{n_{i}}, x_{0}\right)=\phi\left(p^{*}, x_{0}\right)$, which shows that $\lim _{n \rightarrow \infty} \varphi\left(x_{n}, x_{0}\right)=\varphi\left(p^{*}, x_{0}\right)$. If there exists some sequence 


$$
\begin{aligned}
\phi\left(p^{*}, q\right) & =\lim _{n_{i}, n_{j} \rightarrow \infty} \phi\left(x_{n_{i}}, x_{n_{j}}\right)=\lim _{n_{i}, n_{j} \rightarrow \infty} \phi\left(x_{n_{i}}, \Pi_{C_{n_{j}}} x_{0}\right) \\
& \leq \lim _{n_{i}, n_{j} \rightarrow \infty}\left(\phi\left(x_{n_{i}}, x_{0}\right)-\phi\left(\Pi_{C_{n_{j}}} x_{0}, x_{0}\right)\right. \\
& =\lim _{n_{i}, n_{j} \rightarrow \infty}\left(\phi\left(x_{n_{i}}, x_{0}\right)-\phi\left(x_{n_{j}}, x_{0}\right)\right. \\
& =\phi\left(p^{*}, x_{0}\right)-\phi\left(p^{*}, x_{0}\right)=0 .
\end{aligned}
$$

Lemma 1.2(a) we have that

$$
\begin{aligned}
\phi\left(p^{*}, q\right) & =\lim _{n_{i}, n_{j} \rightarrow \infty} \phi\left(x_{n_{i}}, x_{n_{j}}\right)=\lim _{n_{i}, n_{j} \rightarrow \infty} \phi\left(x_{n_{i}}, \Pi_{C_{n_{j}}} x_{0}\right) \\
& \leq \lim _{n_{i}, n_{j} \rightarrow \infty}\left(\phi\left(x_{n_{i}}, x_{0}\right)-\phi\left(\Pi_{C_{n_{j}}} x_{0}, x_{0}\right)\right. \\
& =\lim _{n_{i}, n_{j} \rightarrow \infty}\left(\phi\left(x_{n_{i}}, x_{0}\right)-\phi\left(x_{n_{j}}, x_{0}\right)\right. \\
& =\phi\left(p^{*}, x_{0}\right)-\phi\left(p^{*}, x_{0}\right)=0 .
\end{aligned}
$$

This implies that $p^{*}=q$ and

$$
\lim _{n \rightarrow \infty} x_{n}=p^{*} \text {. }
$$

(V) Now we prove that $p^{*} \in G=\mathscr{F} \cap \Omega$.

First, we prove that $p^{*} \in \mathscr{F}$. In fact, since $x_{n+1} \in C_{n+1} \subset C_{n}$, it follows from (3.1) and (3.6) that

$$
\phi\left(x_{n+1}, y_{n}\right) \leq \phi\left(x_{n+1}, x_{n}\right)+\xi_{n} \rightarrow 0(n \rightarrow \infty) .
$$

By the virtue of Lemma 2.1, we have

$$
\lim _{n \rightarrow \infty} y_{n}=p^{*}
$$

From (3.5), for any $u \in \mathscr{F}$ and $w_{n, i} \in T_{i}^{n} x_{n}$, we have

$$
\phi\left(u, y_{n}\right) \leq \phi\left(u, x_{n}\right)+\xi_{n}-\left(1-\alpha_{n}\right) \beta_{n, 0} \beta_{n, l} g\left(\left\|J x_{n}-J w_{n, l}\right\|\right),
$$

i.e.,

$$
\left(1-\alpha_{n}\right) \beta_{n, 0} \beta_{n, l} g\left(|| J x_{n}-J w_{n, l} \|\right) \leq \phi\left(u, x_{n}\right)+\xi_{n}-\phi\left(u, y_{n}\right) \rightarrow 0(n \rightarrow \infty) .
$$

By conditions (ii) and (iii) it shows that $\lim _{n \rightarrow \infty} g\left(|| J x_{n}-J w_{n, l}||\right)=0$. In view of property of $g$, we have

$$
\left\|J x_{n}-J w_{n, l}\right\| \rightarrow O(n \rightarrow \infty), \forall l \geq 1 .
$$

Since $J x_{n} \rightarrow J p^{*}$, this implies that $J w_{n, l} \rightarrow J p^{*}$. From Remark 1.1 (ii) it yields

$$
w_{n, l} \rightarrow p^{*}(n \rightarrow \infty), \forall l \geq 1 .
$$

Again since

$$
\left\|| w _ { n , l } \| - \| p ^ { * } | \left|=\left\||| J w_{n, l}\right\|-\left\|J p^{*}|| \leq\right\| J w_{n, l}-J p^{*} \| \rightarrow 0(n \rightarrow \infty),\right.\right.
$$

this together with (3.8) and the Kadec-Klee property of $X$ shows that

$$
\lim _{n \rightarrow \infty} w_{n, l}=p^{*}, \forall l \geq 1 \text {. }
$$


Let $\left\{s_{n, l}\right\}$ be a sequence generated by

$$
\begin{array}{r}
s_{2, l} \in T_{l} w_{1, l} \subset T_{l}^{2} x_{1}, s_{3, l} \in T_{l} w_{2, l} \subset T_{l}^{3} x_{2}, \ldots, \\
s_{n+1, l} \in T_{l} w_{n, l} \subset T_{l}^{n+1} x_{n}, \ldots, l \geq 1
\end{array}
$$

By the assumption that each $T_{i}$ is uniformly $L_{i}$-Lipschitz continuous, hence for any $w_{n, l} \in T_{l}^{n} x_{n}$ and $s_{n+1, l} \in T_{l} w_{n} \subset T_{l}^{n+1} x_{n}$ we have

$$
\begin{aligned}
\left\|s_{n+1, l}-w_{n, l}\right\| & \leq\left\|s_{n+1, l}-w_{n+1,1}\right\|+\left\|w_{n+1, l}-x_{n+1}\right\|+\left\|x_{n+1}-x_{n}\right\|+\left\|x_{n}-w_{n, l}\right\| \\
& \leq\left(L_{l}+1\right)\left\|x_{n+1}-x_{n}\right\|+\left\|w_{n+1, l}-x_{n+1}\right\|+\left\|x_{n}-w_{n, l}\right\| .
\end{aligned}
$$

This together with (3.6) and (3.10) shows that

$$
\begin{aligned}
& \lim _{n \rightarrow \infty}\left\|s_{n+1, l}-w_{n, l}\right\|=0, \text { and } \\
& \lim _{n \rightarrow \infty} s_{n+1, l}=p^{*} .
\end{aligned}
$$

In view of the closeness of $T_{l}$, it yields that $p^{*} \in T p^{*}$, i.e., $p^{*} \in F\left(T_{l}\right)$. By the arbitrariness of $l \geq 1$, we have

$$
p^{*} \in \mathscr{F}=\cap_{i=1}^{\infty} F\left(T_{i}\right) .
$$

Next, we prove that $p^{*} \in \Omega$. Since $x_{n+1}=\Pi_{C_{n+1}} x_{0} \in C_{n}$, it follows from (3.1) and (3.6) that

$$
\phi\left(x_{n+1}, u_{n}\right) \leq \phi\left(x_{n+1}, x_{n}\right)+\xi_{n} \rightarrow 0(n \rightarrow \infty) .
$$

Since $x_{n} \rightarrow p^{*}$, by virtue of Lemma 2.1 we have

$$
\lim _{n \rightarrow \infty} u_{n}=p^{*} \text {. }
$$

This together with (3.7) shows that $\left\|u_{n}-y_{n}\right\| \rightarrow 0$ and $\lim _{n \rightarrow \infty}\left\|J u_{n}-J y_{n}\right\| \rightarrow 0$. By the assumption that $r_{n} \geq a, \forall n \geq 0$, we have

$$
\lim _{n \rightarrow \infty} \frac{\left\|J u_{n}-J y_{n}\right\|}{r_{n}}=0
$$

Since $H\left(u_{n}, y\right)+\frac{1}{r_{n}}\left\langle y-u_{n}, J u_{n}-J y_{n}\right\rangle \geq 0, \forall y \in C$, by condition $\left(A_{1}\right)$, we have

$$
\frac{1}{r_{n}}\left\langle y-u_{n}, J u_{n}-J y_{n}\right\rangle \geq-H\left(u_{n}, y\right) \geq H\left(y, u_{n}\right), \forall y \in C \text {. }
$$

By the assumption that $y \mapsto H(x, y)$ is convex and lower semi-continuous, letting $n \rightarrow$ $\infty$ in (3.13), from (3.11) and (3.12), we have $H\left(y, p^{*}\right) \leq 0, \forall y \in C$.

For $t \in(0,1]$ and $y \in C$, letting $y_{t}=t y+(1-t) p^{*}$, therefore $y_{t} \in C$ and $H\left(y_{t}, p^{*}\right) \leq 0$. By condition $\left(A_{1}\right)$ and $\left(A_{4}\right)$, we have

$$
0=H\left(y_{t}, y_{t}\right) \leq t H\left(y_{t}, \gamma\right)+(1-t) H\left(y_{t}, p^{*}\right) \leq t H\left(y_{t}, \gamma\right) .
$$

Dividing both sides of the above equation by $t$, we have $H\left(y_{t}, y\right) \leq 0, \forall y \in C$. Letting $t \downarrow 0$, from condition $\left(A_{3}\right)$, we have $H\left(p^{*}, y\right) \leq 0, \forall y \in C$, i.e., $p^{*} \in \Omega$, and $p^{*} \in \mathscr{G}=\mathscr{F} \cap \Omega$.

(VI) we prove that $x_{n} \rightarrow p^{*}=\prod_{\mathscr{G}} x_{0} .$. 
Let $q=\prod_{G} x_{0}$. Since $q \in \mathscr{G} \subset C_{n}$ and $x_{n}=\prod_{C n} x_{0}$, we have

$$
\phi\left(x_{n}, x_{0}\right) \leq \phi\left(q, x_{0}\right), \quad \forall n \geq 0 .
$$

This implies that

$$
\phi\left(p^{*}, x_{0}\right)=\lim _{n \rightarrow \infty} \phi\left(x_{n}, x_{0}\right) \leq \phi\left(q, x_{0}\right)
$$

In view of the definition of $\prod_{g} x_{0}$, from (3.14) we have $p^{*}=q$. Therefore, $x_{n} \rightarrow p^{*}=\prod_{g} x_{0}$. This completes the proof of Theorem 3.1.

Definition 3.2 A finite family of multi-valued mappings $\left\{T_{i}\right\}_{i=1}^{\infty}: C \rightarrow 2^{C}$ is said to be uniformly quasi- $\varphi$-asymptotically nonexpansive, if $\mathscr{F}=\bigcap_{i=1}^{\infty} F\left(T_{i}\right) \neq \emptyset$ and there exists a real sequence $\left\{k_{n}\right\} \subset[1, \infty), k_{n} \rightarrow 1$ such that for each $i=1,2, \ldots, N$

$$
\phi\left(p, w_{n, i}\right) \leq k_{n} \phi(p, x), \forall x \in C, p \in \bigcap_{i=1}^{\infty} F\left(T_{i}\right), w_{n, i} \in T_{i}^{n} x
$$

The following theorems can be obtained from Theorem 3.1 immediately.

Theorem 3.3 Let $X, C, \Theta, A, \psi$ be as in Theorem 3.1. Let $\left\{T_{i}\right\}_{i=1}^{\infty}$ be a countable family of closed and uniformly quasi- $\varphi$-asymptotically nonexpansive multi-valued mappings with a real sequence $\left\{k_{n}\right\} \subset[1, \infty), k_{n} \rightarrow 1$ and for each $i=1,2, \ldots, T_{i}$ be uniformly $L_{i}$-Lipschitzian. Let $\left\{x_{n}\right\}$ be the sequence generated by

$$
\left\{\begin{array}{l}
x_{0} \in C \text { chosen arbitrary, } C_{0}=C, \\
y_{n}=J^{-1}\left(\alpha_{n} J x_{n}+\left(1-\alpha_{n}\right) J z_{n}\right), \forall n \geq 1, \\
z_{n}=J^{-1}\left(\beta_{n, 0} J x_{n}+\sum_{i=1}^{\infty} \beta_{n, i} J w_{n, i}\right),\left(w_{n, i} \in T_{i}^{n} x_{n}, i \geq 1\right), \forall n \geq 1, \\
u_{n} \in C \text { such that } \forall y \in C \\
\Theta\left(u_{n}, y\right)+\left\langle A u_{n}, y-u_{n}\right\rangle+\psi(y)-\psi\left(u_{n}\right)+\frac{1}{r_{n}}\left\langle y-u_{n} J u_{n}-J y_{n}\right\rangle \geq 0, \\
C_{n+1}=\left\{v \in C_{n}: \phi\left(v, u_{n}\right) \leq \phi\left(v, x_{n}\right)+\xi_{n}\right\}, \quad \forall n \geq 0, \\
x_{n+1}=\Pi_{C_{n+1}} x_{0}, \forall n \geq 0,
\end{array}\right.
$$

where $\mathscr{F}:=\bigcap_{i=1}^{\infty} F\left(T_{i}\right), \xi_{n}=\left(k_{n}-1\right) \sup _{p \in \mathscr{F}} \zeta\left(\phi\left(p, x_{n}\right)\right),\left\{\beta_{n, 0}, \beta_{n, i}\right\}_{i=1}^{\infty}$, and $\left\{\alpha_{n}\right\}$ are sequences in $0[1]$ satisfying the conditions (i), (ii), (iii) in Theorem 3.1. If $\mathscr{F}:=\bigcup_{i=1}^{\infty} F\left(T_{i}\right)$ is a bounded subset of $C$, then $\left\{x_{n}\right\}$ converges strongly to $\prod_{\mathscr{G}} x_{0}$.

Proof. Since $\left\{T_{i}\right\}_{i=1}^{\infty}$ is a countable family of closed and uniformly quasi- $\varphi$-asymptotically nonexpansive multi-valued mappings, by Remark 1.5(2), it is a countable family of closed and uniformly total quasi- $\varphi$-asymptotically nonexpansive multi-valued mappings with non-negative sequences $\left\{v_{n}=\left(k_{n}-1\right)\right\},\left\{\mu_{n}=0\right\}$ and a strictly increasing and continuous function $\zeta(t)=t, t \geq 0$. Hence $\xi_{n}=\left(k_{n}-1\right) \sup _{p \in \mathscr{F}} \phi\left(p, x_{n}\right) \rightarrow 0$ (as $n \rightarrow$ $\infty)$. Therefore all conditions in Theorem 3.1 are satisfied. The conclusion of Theorem 3.3 can be obtained from Theorem 3.1 immediately.

Theorem 3.4 Let $X, C, \Theta, A, \psi$ be as in Theorem 3.1. Let $\left\{T_{i}\right\}_{i=1}^{\infty}$ be a countable family of closed and quasi- $\varphi$-nonexpansive multi-valued mappings. Let $\left\{x_{n}\right\}$ be the sequence generated by 


$$
\left\{\begin{array}{l}
x_{0} \in C \text { chosen arbitrary, } C_{0}=C, \\
y_{n}=J^{-1}\left(\alpha_{n} J x_{n}+\left(1-\alpha_{n}\right) J z_{n}\right), \forall n \geq 1, \\
z_{n}=J^{-1}\left(\beta_{n, 0} J x_{n}+\sum_{i=1}^{\infty} \beta_{n, i} J w_{n, i}\right),\left(w_{n, i} \in T_{i}^{n} x_{n}, i \geq 1\right), \forall n \geq 1, \\
u_{n} \in C \text { such that } \forall y \in C, \forall n \geq 1, \\
\Theta\left(u_{n}, y\right)+\left\langle A u_{n}, y-u_{n}\right\rangle+\psi(y)-\psi\left(u_{n}\right)+\frac{1}{r_{n}}\left\langle y-u_{n}, J u_{n}-J y_{n}\right\rangle \geq 0, \\
C_{n+1}=\left\{v \in C_{n}: \phi\left(v, u_{n}\right) \leq \phi\left(v, x_{n}\right)\right\}, \forall n \geq 0, \\
x_{n+1}=\Pi_{C_{n+1}} x_{0}, \forall n \geq 0,
\end{array}\right.
$$

where $\left\{\beta_{n, 0}, \beta_{n, i}\right\}_{i=1}^{\infty}$ and $\left\{\alpha_{n}\right\}$ are sequences in $0[1]$ satisfying the conditions (i), (ii), (iii) in Theorem 3.1. If $\mathscr{F}:=\bigcap_{i=1}^{\infty} F\left(T_{i}\right) \neq \emptyset$, then $\left\{x_{n}\right\}$ converges strongly to $\Pi_{\mathscr{G}} x_{0}$.

Proof. Since $\left\{T_{i}\right\}_{i=1}^{\infty}$ is a countable family of closed quasi- $\varphi$-nonexpansive multivalued mappings, by Remark 1.5(3), it is a countable of closed and uniformly quasi- $\varphi$ asymptotically nonexpansive multi-valued mappings with sequence $\left\{k_{n}=1\right\}$. Hence $\xi_{n}=\left(k_{n}-1\right) \sup _{u \in \mathscr{F}} \phi\left(u, x_{n}\right)=0$ Therefore, the conditions appearing in Theorem 3.3: " $\mathscr{F}$ is a bounded subset in $C$ " and "for each $i \geq 1, T_{i}$ is uniformly $L_{i}$-Lipschitz" is no use here. Therefore all conditions in Theorem 3.3 are satisfied. The conclusion of Theorem 3.4 can be obtained from Theorem 3.3 immediately.

Remark 3.5 Theorems 3.1, 3.3, and 3.4 not only generalize the corresponding results of Matsushita and Takahashi [5], Plubtieng and Ungchittrakool [6], Ceng et al. [9], Su et al. [10], Ofoedu and Malonza [11], Wang et al. [12], Chang et al. [4,7,8,13,17,19,20], Yao et al. [14], Zegeye et al. [15] and Nilsrakoo and Saejung [16] from single-valued mappings to multi-valued mappings, but also improve and extend the main results of Homaeipour and Razani [3] and the method adopted in this article is also different from that one adopted in [3].

\section{Applications}

In this section, we shall utilize the results presented in Section 3 to study some problems.

\section{(I) Application to convex feasibility problem.}

The "so called" convex feasibility problem for a family of mappings $\left\{T_{i}\right\}_{i=1}^{\omega}$ (where $\omega$ is a finite positive integer or $+\infty$ ) is to finding a point in the nonempty intersection $\bigcap_{i=1}^{\omega} C_{i}$, where $C_{i}$ is a fixed point set of $T_{i}, i=1,2, \ldots, \omega$.

In Theorem 3.4 if $\Theta=0, A=0, \psi=0$, then by Lemma 1.2(c), the condition " $u_{n} \in C$ such that $\forall y \in C,\left\langle y-u_{n}, J u_{n}-J y_{n}\right\rangle \geq 0$ " is equivalent to $u_{n}=\Pi_{C}\left(y_{n}\right)$. Hence from Theorem 3.4 , the iterative sequence $\left\{x_{n}\right\}$ defined by

$$
\left\{\begin{array}{l}
x_{0} \in C \text { chosen arbitrary, } C_{0}=C, \\
y_{n}=J^{-1}\left(\alpha_{n} J x_{n}+\left(1-\alpha_{n}\right) J z_{n}\right), \forall n \geq 1, \\
z_{n}=J^{-1}\left(\beta_{n, 0} J x_{n}+\sum_{i=1}^{\infty} \beta_{n, i} J w_{n, i}\right),\left(w_{n, i} \in T_{i}^{n} x_{n}, i \geq 1\right), \forall n \geq 1, \\
C_{n+1}=\left\{v \in C_{n}: \phi\left(v, u_{n}\right) \leq \phi\left(v, x_{n}\right)\right\}, u_{n}=\Pi_{C} y_{n}, \quad \forall n \geq 1 \\
x_{n+1}=\Pi_{C_{n+1}} x_{0}, \forall n \geq 0
\end{array}\right.
$$


converges strongly to a point $p^{*}=\prod_{\mathscr{F}} x_{0}$, which is a solution of the convex feasibility problem for a countable family of closed and quasi- $\varphi$-nonexpansive multi-valued mappings $\left\{T_{i}\right\}_{i=1}^{\infty}$ where $\mathscr{F}=\bigcap_{i=1}^{\infty} F\left(T_{i}\right)$.

(II) Application to generalized MEP

In Theorem 3.4 taking $T_{i}=I, \forall i \geq 1$, (the identity mapping on $C$ ), then $z_{n}=y_{n}=x_{n}, \forall n \geq 1, \mathscr{F}=C, \mathscr{G}=\Omega$. By Theorem 3.4 the sequence $\left\{x_{n}\right\}$ defined by

$$
\left\{\begin{array}{l}
x_{0} \in C \text { chosen arbitrary, } \quad C_{0}=C, \\
u_{n} \in C \text { such that } \forall y \in C, \forall n \geq 1, \\
\Theta\left(u_{n}, y\right)+\left\langle A u_{n}, y-u_{n}\right\rangle+\psi(\gamma)-\psi\left(u_{n}\right)+\frac{1}{r_{n}}\left\langle y-u_{n}, J u_{n}-J x_{n}\right\rangle \geq 0, \\
C_{n+1}=\left\{v \in C_{n}: \phi\left(v, u_{n}\right) \leq \phi\left(v, x_{n}\right)\right\}, \quad \forall n \geq 0, \\
x_{n+1}=\Pi_{C_{n+1}} x_{0}, \quad \forall n \geq 0 .
\end{array}\right.
$$

converges strongly to a point $p^{*}=\Pi_{\Omega} x_{0}$, which is a solution of the generalized MEP (1.1).

\section{(III) Application to optimization problem}

In (4.2), if $\Theta=0, A=0$, then from Theorem 3.4 the sequence $\left\{x_{n}\right\}$ defined by

$$
\left\{\begin{array}{l}
x_{0} \in C \text { chosen arbitrary, } C_{0}=C, \\
u_{n} \in C \text { such that } \forall y \in C \\
\psi(y)-\psi\left(u_{n}\right)+\frac{1}{r_{n}}\left\langle y-u_{n}, J u_{n}-J x_{n}\right\rangle \geq 0 \\
C_{n+1}=\left\{v \in C_{n}: \phi\left(v, u_{n}\right) \leq \phi\left(v, x_{n}\right)\right\}, \quad \forall n \geq 0 \\
x_{n+1}=\Pi_{C_{n+1}} x_{0} .
\end{array}\right.
$$

converges strongly to a point $p^{*}=\prod_{K} x_{0}$ which is a solution of the optimization problem $\min _{x \downarrow} C \psi(x)$, where $K \subset C$ is the set of solutions to this optimization problem.

(IV) Application to the mixed variational inequality problem of Browder type In (4.2), if $\Theta=0$, then the iterative sequence $\left\{x_{n}\right\}$ defined by

$$
\left\{\begin{array}{l}
x_{0} \in C \text { chosen arbitrary, } C_{0}=C, \\
u_{n} \in C \text { such that } \forall y \in C \\
\left\langle A u_{n}, y-u_{n}\right\rangle+\psi(y)-\psi\left(u_{n}\right)+\frac{1}{r_{n}}\left\langle y-u_{n}, J u_{n}-J x_{n}\right\rangle \geq 0, \\
C_{n+1}=\left\{v \in C_{n}: \phi\left(v, u_{n}\right) \leq \phi\left(v, x_{n}\right)\right\}, \quad \forall n \geq 0, \\
x_{n+1}=\Pi_{C_{n+1}} x_{0} .
\end{array}\right.
$$

converges strongly to a point $p^{*}=\prod_{Q} x_{0}$ which is a solution of the mixed variational inequality of Browder type (1.4), where $Q$ is the set of solutions to equation (1.4).

${ }^{1}$ College of Statistics and Mathematics, Yunnan University of Finance and Economics, Kunming, Yunnan 650221, China ${ }^{2}$ School of Information Engineering, the College of Arts and Sciences, Yunnan Normal University, Kunming, Yunnan 650222, China 


\section{Competing interests}

The authors declare that they have no competing interests.

Received: 16 January 2012 Accepted: 30 April 2012 Published: 30 April 2012

\section{References}

1. Cioranescu, I: Geometry of Banach Spaces, Duality Mappings and Nonlinear Problems. Kluwer Academic Publishers, Dordrecht. (1990)

2. Alber, YI: Metric and generalized projection operators in Banach space: properties and application. In: Kartosator AG (ed.) Theory and Applications of Nonlinear Operators of Accretive and Monotone Type. pp. 15-50. Marcel Dekker, New York (1996)

3. Homaeipour, S, Razani, A: Weak and strong convergence theorems for relatively nonexpansive multi-valued mappings in Banach spaces. Fixed Point Theorem Appl 73 (2011). doi:10.1186/1687-1812-2011-73

4. Chang, S-s, Joseph Lee, HW, Chi Kin, Chan, Zhang, WB: A modified Halpern-type iterative algorithm for totally quasi- $₫$ asymptotically nonexpansive mappings with applications. Appl Math Comput. 218, 6489-6497 (2012). doi:10.1016/j. amc.2011.12.019

5. Matsushita, S, Takahashi, W: A strong convergence theorem for relatively nonexpansive mappings in Banach spaces. J Approx Theory. 134, 257-266 (2005). doi:10.1016/j.jat.2005.02.007

6. Plubtieng, S, Ungchittrakool, K: Hybrid iterative method for convex feasibility problems and fixed point problems of relatively nonexpansive mappings in Banach spaces. Fixed Point Theor Appl 2008, 19 (2008). Article ID 583082, doi:10.1155/2008/58308

7. Chang, S-s, Joseph Lee, HW, Chan, CK: A block hybrid method for solving generalized equilibrium problems and convex feasibility problem. Adv Comput Math (2011). doi:10.1007/s10444-011-9249-5

8. Chang, S-s, Joseph Lee, HW, Chan, CK, Yang, L: Approximation theorems for total quasi-囚-asymptotically nonexpansive mappings with applications. Appl Math Comput. 218, 2921-2931 (2011). doi:10.1016/j.amc.2011.08.036

9. Ceng, L-C, Guu, S-M, Hu, H-Y, Yao, J-C: Hybrid shrinking projection method for a generalized equilibrium problem, a maximal monotone operator and a countable family of relatively nonexpansive mappings. Comput Math Appl. 61, 2468-2479 (2011). doi:10.1016/j.camwa.2011.02.028

10. Su, YF, Xu, HK, Zhang, X: Strong convergence theorems for two countable families of weak relatively nonexpansive mappings and applications. Nonlinear Anal. 73, 3890-3906 (2010). doi:10.1016/j.na.2010.08.021

11. Ofoedu, EU, Malonza, DM: Hybrid approximation of solutions of nonlinear operator equations and application to equation of Hammerstein-type. Appl Math Comput. 217, 6019-6030 (2011). doi:10.1016/j.amc.2010.12.073

12. Wang, ZM, Su, YF, Wang, DX, Dong, YC: A modified Halpern-type iteration algorithm for a family of hemi-relative nonexpansive mappings and systems of equilibrium problems in Banach spaces. J Comput Appl Math. 235, 2364-2371 (2011). doi:10.1016/j.cam.2010.10.036

13. Chang, SS, Chan, CK, Joseph Lee, HW: Modified block iterative algorithm for quasi-囚-asymptotically nonexpansive mappings and equilibrium problem in Banach spaces. Appl Math Comput. 217, 7520-7530 (2011). doi:10.1016/j. amc.2011.02.060

14. Yao, YH, Liou, YC, Kang, SM: Strong convergence of an iterative algorithm on an infinite countable family of nonexpansive mappings. Appl Math Comput. 208, 211-218 (2009). doi:10.1016/j.amc.2008.11.038

15. Zegeye, H, Ofoedu, EU, Shahzad, N: Convergence theorems for equilibrium problem, variational inequality problem and countably infinite relatively quasi-nonexpansive mappings. Appl Math Comput. 216, 3439-3449 (2010). doi:10.1016/j. amc.2010.02.054

16. Nilsrakoo, W, Saejung, S: Strong convergence theorems by Halpern-Mann iterations for relatively non-expansive mappings in Banach spaces. Appl Math Comput. 217, 6577-6586 (2011). doi:10.1016/j.amc.2011.01.040

17. Chang, S-S, Joseph Lee, HW, Chan, CK, Liu, Ja: Strong convergence theorems for countable families of asymptotically relatively nonexpansive mappings with applications. Appl Math Comput. 218, 3187-3198 (2011). doi:10.1016/j. amc.2011.08.055

18. Zhang, S-s: The generalized mixed equilibrium problem in Banach space. Appl Math Mech. 30, 1105-1112 (2009). doi:10.1007/s10483-009-0904-6

19. Chang, S-s, Kim, JK, Wang, XR: Modified block iterative algorithm for solving convex feasibility problems in Banach spaces. J Inequal Appl 2010, V (2010). Article ID 869684 (2010) 14 pages, doi: 1155/2010/869684. doi:10.1155/2010/ 869684

20. Chang, S-s, Joseph Lee, HW, Chan, CK, Yang, L: Approximation theorems for total quasi-囚-asymptotically nonexpansive mappings with applications. Appl Math Comput. 218, 2921-2931 (2011). doi:10.1016/j.amc.2011.08.036

21. Luchuan, C, Jenchih, Y: A hybrid iterative scheme for mixed equilibrium problems and fixed point problems. J Comput Appl Math. 214, 186-201 (2008). doi:10.1016/j.cam.2007.02.022

doi:10.1186/1687-1812-2012-69

Cite this article as: Chang et al: Strong convergence theorems of nonlinear operator equations for countable family of multi-valued total quasi- $\varphi$-asymptotically nonexpansive mappings with applications. Fixed Point Theory and Applications 2012 2012:69. 